\title{
SWITCHING NEAR A NETWORK OF ROTATING NODES
}

\author{
MANUELA A.D. AGUIAR, ISABEL S. LABOURIAU, \\ AND ALEXANDRE A. P. RODRIGUES
}

\begin{abstract}
We study the dynamics of a $\mathbf{Z}_{2} \oplus \mathbf{Z}_{2}$ - equivariant vector field in the neighbourhood of a heteroclinic network with a periodic trajectory and symmetric equilibria. We assume that around each equilibrium the linearization of the vector field has non-real eigenvalues. Trajectories starting near each node of the network turn around in space either following the periodic trajectory or due to the complex eigenvalues near the equilibria. Thus, a network with rotating nodes. The rotations combine with transverse intersections of two-dimensional invariant manifolds to create switching near the network: close to the network there are trajectories that visit neighbourhoods of the saddles following all the heteroclinic connections of the network in any given order. Our results are motivated by an example where switching was observed numerically, by forced symmetry breaking of an asymptotically stable network with $\mathbf{O}(2)$ symmetry.
\end{abstract}

\section{INTRODUCTION}

Heteroclinic connections and networks are a common feature of symmetric differential equations, and persist under perturbations that preserve the symmetry. Start with an asymptotically stable network with $\mathbf{O}(2)$ symmetry. A perturbation that breaks part of the symmetry splits a two-dimensional connection into a pair of one-dimensional ones. The new network is no longer asymptotically stable, nearby trajectories follow the network around in a complex way that we call switching.

By a heteroclinic network we mean a connected flow-invariant set that is the union of heteroclinic cycles. In the present case it is the orbit under the symmetry group $\mathbf{Z}_{2} \oplus \mathbf{Z}_{2}$ of a heteroclinic cycle. These

2000 Mathematics Subject Classification. Primary: 37G30; Secondary: 37C10, 34C37, 37C29, 34C28, 37C80.

Key words and phrases. heteroclinic network, switching, vector fields, symmetry breaking, shadowing.

The research of all authors at Centro de Matemática da Universidade do Porto (CMUP) had financial support from Fundação para a Ciência e a Tecnologia (FCT), Portugal, through the programs POCTI and POSI with European Union and national funding. A.A.P. Rodrigues was supported by the grant $\mathrm{SFRH} / \mathrm{BD} / 28936 / 2006$ of FCT. 
networks are often called heteroclinic cycles in the literature. Heteroclinic cycles and networks are known to occur persistently in the settings of symmetry [8], [16], coupled cell systems (with and without symmetry) [6], [2] and population dynamics [12], [13], [11], [7]. They are induced by the existence of flow invariant subpaces that correspond, respectively, to fixed point subspaces, synchrony subspaces and coordinate axes and hyperplanes.

We study the dynamics near such a network where all cycles have a common node that is a closed trajectory. We prove that there are trajectories near the network that follow its cycles in any desired order. Trajectories that go near the periodic orbit may switch to any heteroclinic cycle, return and switch again.

It is worthwhile to isolate general properties that entail switching, so the results may be applied to examples in other contexts.

There exist in the literature several numerical reports on complicated dynamics near heteroclinic networks of equilibria and of equilibria and periodic trajectories, that include random visits to the nodes of the network in any possible order [10], [8], [5], [23].

This type of behaviour is not possible around asymptotically stable heteroclinic networks whose connections are contained in invariant subspaces. Each cycle in the network cannot be asymptotically stable but it may have strong attractivity properties [19], [24] so that each nearby trajectory outside the invariant subspaces will tend to one of the cycles in the network.

Different forms of switching have been described in several contexts. Networks where all the nodes are equilibria, have been studied by Postlethwaite and Dawes [21] who found trajectories that follow three cycles in a network sequentially, both regularly and irregularly; by Kirk and Silber [15] near a network with two cycles who found nearby trajectories that switch in one direction. Persistent random switching is found by Guckenheimer and Worfolk [10] and Aguiar et al [4]; noise induced switching in Armbruster et al [5].

A problem similar to ours, where a network involves equilibria and a periodic trajectory, appears in the heteroclinic model of the geodynamo derived in Melbourne et al [20]. Starting with a model with $\mathbf{Z}_{2} \oplus$ $\mathbf{Z}_{2} \oplus \mathbf{S O}(2)$ symmetry, they perturb the model so the only remaining symmetry is $-I d$. For the perturbed model they establish switching numerically in terms of reversals and excursions.

This has motivated Kirk and Rucklidge [14] to ask whether switching would be observed when all the symmetries are broken. First they analyse partially broken symmetries in two different ways: when only the $\mathbf{S O}(2)$ symmetry remains they find a weak form of switching, where trajectories starting near one equilibrium may visit the neighbourhood of another but not return to the first one; for the $\mathbf{Z}_{2} \oplus \mathbf{Z}_{2}$ symmetric case they find attracting periodic trajectories and no switching. 
Then they argue that when all symmetries are broken and the network is destroyed, switching will not take place arbitrarily close to $\mathbf{Z}_{2} \oplus \mathbf{Z}_{2}$-symmetric problems because of barriers formed by invariant manifolds. They describe a scenario where switching may arise, if the symmetry-breaking terms are larger than a threshold value. They propose a mechanism for switching arising from the right combination of homoclinic tangencies between the stable and unstable manifolds of a periodic orbit and specific heteroclinic tangencies between stable and unstable manifolds of the equilibria.

Here we analyse equations with a symmetry group $\mathbf{Z}_{2} \oplus \mathbf{Z}_{2}$ a subgroup of that considered by Melbourne et al [20] but not acting in the same way as in Kirk and Rucklidge [14]: each $\mathbf{Z}_{2}$ in our setting contains a rotation by $\pi$ that fixes a plane. A discussion of how our results compare with those of [14] and [20] appears at the end of this paper in section 9 .

Under generic hypotheses for this symmetry, we prove a strong form of switching: the existence of trajectories that visit neighbourhoods of any sequence of nodes of the network in any order that is compatible with the network connections.

The conditions we need for switching are stated in section 3 preceded by definitions and preliminary results in section 2 .

In section 4 we present an example of a $\mathbf{Z}_{2} \oplus \mathbf{Z}_{2^{-}}$equivariant family of ordinary differential equations having a network of rotating nodes. When one of the parameters is set to zero, the equations are $\mathbf{Z}_{2} \oplus \mathbf{Z}_{2} \oplus$ SO(2)-symmetric and the network is asymptotically stable. Switching occurs for all small non-zero values of this symmetry-breaking parameter.

We linearize the flow around the invariant saddles in section 5, obtaining isolating blocks around each node of the network. This section is mostly concerned with introducing the notation for the proof of switching that occupies the rest of the paper.

The goal of this paper is to prove switching in the neighbourhood of a heteroclinic network that consists of four symmetric copies of a heteroclinic cycle

$$
\mathrm{C} \rightarrow \mathrm{v} \rightarrow \mathrm{w} \rightarrow \mathrm{C}
$$

where $\mathbf{C}$ is a closed trajectory invariant under the symmetries and $\mathbf{v}$ and $\mathbf{w}$ are equilibria. The connection $\mathbf{v} \rightarrow \mathbf{w}$ is one-dimensional and takes place inside a fixed-point subspace, the other connections are transverse intersections of 2-dimensional invariant manifolds. The trajectory $\mathbf{C}$ has real Floquet multipliers and 2-dimensional stable and unstable manifolds; the linearisation of the flow near $\mathbf{v}$ has a pair of complex eigenvalues with positive real part and one real negative eigenvalue; the linearisation of the flow near $\mathbf{w}$ has one real positive eigenvalue and pair of complex eigenvalues with negative real part (see figures 1 and 2). 
In section 6 we obtain a geometrical description of the way the flow transforms a curve of initial conditions lying across the stable manifold of each node. The curve is wrapped around the isolating block of the next node, accumulating on its unstable manifold and in particular on the next connection. Thus, points on a line across the stable manifold of $\mathbf{v}$ will be mapped into a helix accumulating on the unstable manifold of $\mathbf{w}$ that will cross the transverse stable manifold of $\mathbf{C}$ infinitely many times. Similarly, points on a line across the stable manifold of $\mathbf{C}$ will be mapped into a helix accumulating on its unstable manifold and thus will cross the transverse stable manifold of $\mathbf{v}$ infinitely many times.

The geometrical setting is explored in section 7 to obtain intervals on the curve of initial conditions that are mapped by the flow into curves near the next node in a position similar to the first one. This allows us to establish the recurrence needed for switching in section 8: for any sequence of nodes like

$$
+\mathbf{v} \rightarrow-\mathbf{w} \rightarrow \mathrm{C} \rightarrow-\mathbf{v} \rightarrow-\mathbf{w} \rightarrow \mathrm{C} \rightarrow+\mathbf{v} \rightarrow+\mathbf{w} \rightarrow \mathrm{C} \rightarrow+\mathbf{v} \rightarrow \cdots
$$

we find a trajectory that visits neighbourhoods of these nodes in the same sequence.

We end the paper with a discussion (section 9) of the results obtained and of related results in the literature.

\section{Preliminaries}

Let $f$ be a smooth vector field on $\mathbf{R}^{n}$ with flow given by the unique solution $x(t)=\varphi\left(t, x_{0}\right) \in \mathbf{R}^{n}$ of

$$
\dot{x}=f(x) \quad x(0)=x_{0} .
$$

If $A$ is a compact invariant set for the flow of $f$, we say, following Field [8], that $A$ is an invariant saddle if

$$
A \subseteq \overline{W^{s}(A) \backslash A} \text { and } A \subseteq \overline{W^{u}(A) \backslash A},
$$

where $\bar{A}$ is the closure of $A$. In this paper all the saddles are hyperbolic.

Given two invariant saddles $A$ and $B$, an $m$-dimensional heteroclinic connection from $A$ to $B$, denoted $[A \rightarrow B]$, is an $m$-dimensional connected flow-invariant manifold contained in $\left.W^{u} A\right) \cap W^{s}(B)$. There may be more than one connection from $A$ to $B$.

Let $\mathcal{S}=\left\{A_{j}: j \in\{1, \ldots, k\}\right\}$ be a finite ordered set of mutually disjoint invariant saddles. Following Field [8], we say that there is a heteroclinic cycle associated to $\mathcal{S}$ if

$$
\forall j \in\{1, \ldots, k\}, W^{u}\left(A_{j}\right) \cap W^{s}\left(A_{j+1}\right) \neq \emptyset \quad(\bmod k) .
$$

We refer to the saddles defining the heteroclinic cycle as nodes.

A heteroclinic network is a finite connected union of heteroclinic cycles.

Let $\Gamma$ be a compact Lie group acting linearly on $\mathbf{R}^{n}$. The vector field $f$ is $\Gamma$-equivariant if for all $\gamma \in \Gamma$ and $x \in \mathbf{R}^{n}$, we have $f(\gamma x)=$ 
$\gamma f(x)$. In this case $\gamma \in \Gamma$ is said to be a symmetry of $f$. We refer the reader to Golubitsky, Stewart and Schaeffer [9] for more information on differential equations with symmetry.

The $\Gamma$-orbit of $x_{0} \in \mathbf{R}^{n}$ is the set $\Gamma\left(x_{0}\right)=\left\{\gamma x_{0}, \gamma \in \Gamma\right\}$ that is invariant under the flow of $\Gamma$-equivariant vector fields $f$. In particular, if $x_{0}$ is an equilibrium of (1), so are the elements in its $\Gamma$-orbit.

The isotropy subgroup of $x_{0} \in \mathbf{R}^{n}$ is $\Gamma_{x_{0}}=\left\{\gamma \in \Gamma, \quad \gamma x_{0}=x_{0}\right\}$. For an isotropy subgroup $\Sigma$ of $\Gamma$, its fixed-point subspace is

$$
F i x(\Sigma)=\left\{x \in \mathbf{R}^{n}: \forall \gamma \in \Sigma, \gamma x=x\right\} .
$$

Fixed-point subspaces are the reason for the robustness of heteroclinic cycles and networks in symmetric dynamics: if $f$ is $\Gamma$-equivariant then $\operatorname{Fix}(\Sigma)$ is flow-invariant, thus connections occurring inside these spaces persist under perturbations that preserve the symmetry.

For a heteroclinic network $\Sigma$ with node set $\mathcal{A}$, a path of order $k$, on $\Sigma$ is a finite sequence $s^{k}=\left(c_{j}\right)_{j \in\{1, \ldots, k\}}$ of connections $c_{j}=\left[A_{j} \rightarrow B_{j}\right]$ in $\Sigma$ such that $A_{j}, B_{j} \in \mathcal{A}$ and $B_{j}=A_{j+1}$ i.e. $c_{j}=\left[A_{j} \rightarrow A_{j+1}\right]$. For an infinite path, take $j \in \mathbf{N}$.

Let $N_{\Sigma}$ be a neighbourhood of the network $\Sigma$ and let $U_{A}$ be a neighbourhood of each node $A$ in $\Sigma$. For each heteroclinic connection in $\Sigma$, consider a point $p$ on it and a small neighbourhood $V$ of $p$. We are assuming that the neighbourhoods of the nodes are pairwise disjoint, as well for those of points in connections.

Given neighbourhoods as above, the point $q$, or its trajectory $\varphi(t)$, follows the finite path $s^{k}=\left(c_{j}\right)_{j \in\{1, \ldots, k\}}$ of order $k$, if there exist two monotonically increasing sequences of times $\left(t_{i}\right)_{i \in\{1, \ldots, k+1\}}$ and $\left(z_{i}\right)_{i \in\{1, \ldots, k\}}$ such that for all $i \in\{1, \ldots, k\}$, we have $t_{i}<z_{i}<t_{i+1}$ and:

(1) $\varphi(t) \subset N_{\Sigma}$ for all $t \in\left(t_{1}, t_{k+1}\right)$;

(2) $\varphi\left(t_{i}\right) \in U_{A_{i}}$ and $\varphi\left(z_{i}\right) \in V_{i}$ and

(3) for all $t \in\left(z_{i}, z_{i+1}\right), \varphi(t)$ does not visit the neighbourhood of any other node except that of $A_{i+1}$.

There is finite switching near $\Sigma$ if for each finite path there is a trajectory that follows it. Analogously, we define infinite switching near $\Sigma$ by requiring that each infinite path is followed by a trajectory.

An infinite path on $\Sigma$ may also be seen as a pseudo-orbit of $\dot{x}=f(x)$, with infinitely many discontinuities. Switching near $\Sigma$ means that these infinite pseudo-orbits can be shadowed.

\section{A NETWORK OF ROTATING NODES}

Our object of study is the dynamics around a special type of heteroclinic network (see figure 2) for which we give a rigorous description here. The network lies in a topological three-sphere and one of its nodes is a closed trajectory with real Floquet multipliers and 2-dimensional stable and unstable manifolds. Near this node, the flow rotates following the closed trajectory. The other nodes are equilibria with a pair 

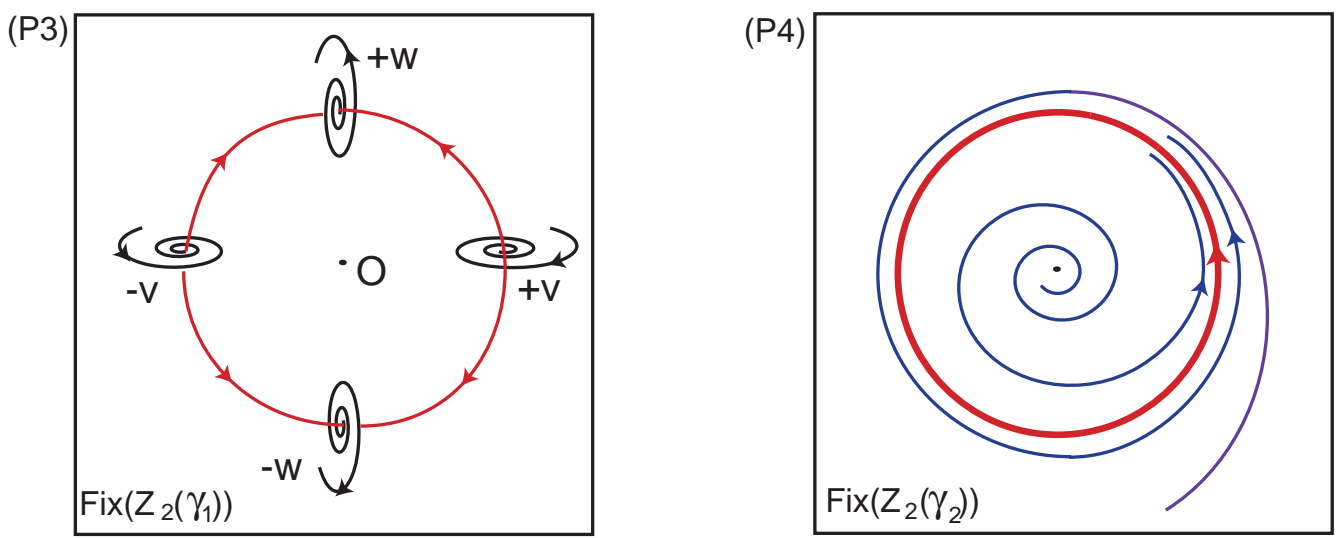

FiguRE 1. Dynamics near a heteroclinic network of rotating nodes. Left: dynamics around the plane Fix $\left(\mathbf{Z}_{2}\left(\gamma_{1}\right)\right)$ illustrating property (P3); Right: dynamics in the plane $\operatorname{Fix}\left(\mathbf{Z}_{2}\left(\gamma_{2}\right)\right)$ illustrating property $(\mathrm{P} 4)$.

of non-real eigenvalues. Thus the local dynamics rotates around each node.

Specifically, we study a smooth vector field $f$ on $\mathbf{R}^{4}$ with the following properties:

(P1) The vector field $f$ is equivariant under $\Gamma \cong \mathbf{Z}_{2} \oplus \mathbf{Z}_{2}$ acting linearly on $\mathbf{R}^{4}$ with generators $\gamma_{1}$ and $\gamma_{2}$ and with two transverse twodimensional fixed-point subspaces. In particular, the origin is an equilibrium.

(P2) There is a three-dimensional flow-invariant manifold $\mathbf{S}^{3}$ diffeomorphic to a sphere that attracts all the trajectories except the origin. For simplicity, we assume this manifold to be the unit sphere.

By (P1-P2) there are two flow-invariant circles

$$
\mathbf{C}_{1}=\mathbf{S}^{3} \cap \operatorname{Fix}\left(\mathbf{Z}_{2}\left(\gamma_{1}\right)\right) \text { and } \mathbf{C}=\mathbf{S}^{3} \cap \operatorname{Fix}\left(\mathbf{Z}_{2}\left(\gamma_{2}\right)\right) .
$$

(P3) On $\mathbf{C}_{1}$ there are exactly four equilibria that will be denoted by $+\mathbf{v},+\mathbf{w},-\mathbf{v}=\gamma_{2} \cdot \mathbf{v},-\mathbf{w}=\gamma_{2} \cdot \mathbf{w}$. Moreover, the eigenvalues of $d f$ restricted to $T \mathbf{S}^{3}$ are:

- $-C_{v} \pm i$ and $E_{v}$ with $C_{v} \neq E_{v}>0$, at $\pm \mathbf{v}$;

- $E_{w} \pm i$ and $-C_{w}$ with $C_{w} \neq E_{w}>0$, at $\pm \mathbf{w}$.

In Fix $\left(\mathbf{Z}_{2}\left(\gamma_{1}\right)\right)$ the equilibria $\pm \mathbf{v}$ are saddles and $\pm \mathbf{w}$ are sinks with connections in $\mathbf{C}_{1}$ from $\pm \mathbf{v}$ to $\pm \mathbf{w}$. These connections are persistent under perturbations that preserve the $\gamma_{1}$-symmetry (see figure 1 ). 
(P4) In the invariant plane $\operatorname{Fix}\left(\mathbf{Z}_{2}\left(\gamma_{2}\right)\right)$ the only equilibrium is the origin and it is an unstable focus.

Thus $\mathbf{C}$ is a closed trajectory and, from (P2), this trajectory is a sink in Fix $\left(\mathbf{Z}_{2}\left(\gamma_{2}\right)\right.$ ) (see figure 1). Since $\mathbf{C}$ is contained in the flow-invariant plane Fix $\left(\mathbf{Z}_{2}\left(\gamma_{2}\right)\right)$, its Floquet multipliers are real.

(P5) The periodic trajectory $\mathbf{C}$ is hyperbolic and, in $\mathbf{S}^{3}, \operatorname{dim} W^{u}(\mathbf{C})=$ $\operatorname{dim} W^{s}(\mathbf{C})=2$. Moreover, there are connections $[\mathbf{C} \rightarrow+\mathbf{v}]$ and $[+\mathbf{w} \rightarrow \mathbf{C}]$ satisfying:

$$
W^{u}(\mathbf{C}) \pitchfork W^{s}(+\mathbf{v}) \text { and } \quad W^{u}(+\mathbf{w}) \pitchfork W^{s}(\mathbf{C}) .
$$

These intersections are one-dimensional and consist of one pair of $\gamma_{1^{-}}$ related trajectories.

From the $\gamma_{2}-$ symmetry, we obtain a pair of $\gamma_{1}$-related one-dimensional connections in $W^{u}(\mathbf{C}) \pitchfork W^{s}(-\mathbf{v})$ and in $W^{u}(-\mathbf{w}) \pitchfork W^{s}(\mathbf{C})$. It follows that there is a heteroclinic network $\Sigma$ involving the saddles $\pm \mathbf{v}$, $\pm \mathbf{w}$ and $\mathbf{C}$ (figure 2). Such a network $\Sigma$ is what we call a network of rotating nodes. This paper shows switching near this network.

The symmetry $\gamma_{1}$ and its flow invariant fixed point subspace ensure the persistence of the connections $[ \pm \mathbf{v} \rightarrow \pm \mathbf{w}]$. The other symmetry $\gamma_{2}$ is not essential for the existence of a robust network with these properties but it makes them more natural as illustrated by the example in section 4 . The same is true for the existence of the invariant 3-sphere. In section 9 we discuss variants of these hypotheses for which switching may be proved in the same way. Note that if the invariant manifolds $W^{u}(\mathbf{C})$ and $W^{s}(-\mathbf{v})$ did not intersect at all, one of them might form a barrier in $\mathbf{S}^{3}$, contradicting some of the other hypotheses.

\section{EXAMPLE}

Our study was initially motivated by the following example constructed in Aguiar [1], using the methods of [3]. This is an ordinary differential equation in $\mathbf{R}^{4}$ given by:

$$
\left\{\begin{array}{l}
\dot{x}_{1}=x_{1}\left(\alpha_{1}+\alpha_{2} r_{1}^{2}+\alpha_{3} x_{3}^{2}+\alpha_{4} x_{4}^{2}+\alpha_{5}\left(x_{3}^{4}-r_{1}^{2} x_{4}^{2}\right)\right)-x_{2} \\
\dot{x}_{2}=x_{2}\left(\alpha_{1}+\alpha_{2} r_{1}^{2}+\alpha_{3} x_{3}^{2}+\alpha_{4} x_{4}^{2}+\alpha_{5}\left(x_{3}^{4}-r_{1}^{2} x_{4}^{2}\right)\right)+x_{1} \\
\dot{x}_{3}=x_{3}\left(\alpha_{1}+\alpha_{2} x_{3}^{2}+\alpha_{3} x_{4}^{2}+\alpha_{4} r_{1}^{2}+\alpha_{5}\left(x_{4}^{4}-r_{1}^{2} x_{3}^{2}\right)\right)+\xi h_{1}(x) \\
\dot{x}_{4}=x_{4}\left(\alpha_{1}+\alpha_{2} x_{4}^{2}+\alpha_{3} r_{1}^{2}+\alpha_{4} x_{3}^{2}+\alpha_{5}\left(r_{1}^{4}-x_{3}^{2} x_{4}^{2}\right)\right)-\xi h_{2}(x)
\end{array}\right.
$$

where $x=\left(x_{1}, x_{2}, x_{3}, x_{4}\right) \in \mathbf{R}^{4}, r_{1}^{2}=x_{1}^{2}+x_{2}^{2}$ and

$h_{1}(x)=\left[\alpha_{1}+3 \alpha_{2}\left(x_{1}^{2}+x_{2}^{2}\right)\right] x_{1} x_{2} x_{4} \quad$ and $\quad h_{2}(x)=\left[\alpha_{1}+3 \alpha_{2}\left(x_{1}^{2}+x_{2}^{2}\right)\right] x_{1} x_{2} x_{3}$.

The symmetries of the equation are changes of sign of pairs of coordinates:

$$
\gamma_{1}(x)=\left(-x_{1},-x_{2}, x_{3}, x_{4}\right) \quad \gamma_{2}(x)=\left(x_{1}, x_{2},-x_{3},-x_{4}\right) .
$$




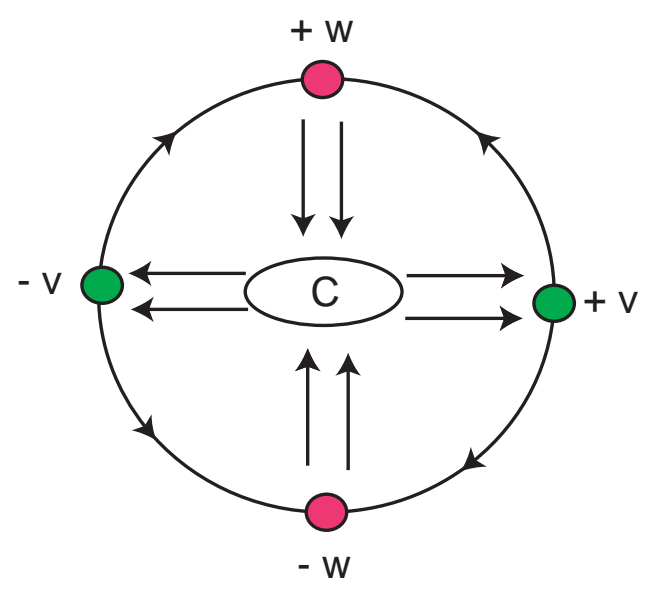

FiguRE 2. Schematic description of a heteroclinic network of rotating nodes satisfying (P1)-(P5). Each arrow represents a 1-dimensional heteroclinic connection. There is one 1-dimensional heteroclinic connection from each equilibrium $\pm \mathbf{v}$ to each equilibrium $\pm \mathbf{w}$ (P3). There are two 1-dimensional heteroclinic connection involving each equilibrium and the periodic trajectory $\mathbf{C}$ (P5).

In [1] it is proved that, for parameter values such that

$$
\begin{gathered}
\alpha_{1}>0 \quad \alpha_{3}+\alpha_{4}=2 \alpha_{2} \quad \alpha_{3}<\alpha_{2}<\alpha_{4}<0 \\
\alpha_{2}\left(\alpha_{3}-\alpha_{4}\right)+\alpha_{1} \alpha_{5}>0
\end{gathered}
$$

and if $\xi \geq 0$ is such that

$\xi<\frac{-\alpha_{2} \alpha_{3}+\alpha_{2} \alpha_{4}+\alpha_{1} \alpha_{5}}{2 \alpha_{1} \alpha_{2}}$ and $\xi^{2}<\frac{\left(\alpha_{4}-\alpha_{3}\right)\left(2 \alpha_{1} \alpha_{5}-\alpha_{2} \alpha_{3}+\alpha_{2} \alpha_{4}\right)}{4 \alpha_{1}^{2} \alpha_{2}}$

then its dynamics satisfies (P1-P4) as we proceed to explain.

For $\xi=0$ the equation has more symmetry, like the model in Melbourne et al [20]: it is equivariant under the group $\mathbf{Z}_{2} \oplus \mathbf{Z}_{2} \oplus \mathbf{S O}(2)$, generated by

$$
\begin{aligned}
& \kappa_{\varphi}(x)=\left(x_{1} \cos (\varphi)-x_{2} \sin (\varphi), x_{1} \sin (\varphi)+x_{2} \cos (\varphi), x_{3}, x_{4}\right) \\
& \kappa_{2}(x)=\left(x_{1}, x_{2},-x_{3}, x_{4}\right) \quad \kappa_{3}(x)=\left(x_{1}, x_{2}, x_{3},-x_{4}\right) .
\end{aligned}
$$

For $\xi=0$ the three-dimensional sphere $\mathbf{S}_{r}^{3}$ of radius $r=\sqrt{\frac{-\alpha_{1}}{\alpha_{2}}}$ is flow invariant and globally attracting. The equilibria $\pm \mathbf{v}$ (resp. $\pm \mathbf{w}$ ) lie at the intersection of $\mathbf{S}_{r}^{3}$ with line fixed by the subgroup generated by $\kappa_{\varphi}$ and $\kappa_{2}$ (resp. $\kappa_{\varphi}$ and $\kappa_{3}$ ). The closed trajectory $\mathbf{C}$ is the intersection of $\mathbf{S}_{r}^{3}$ with the plane fixed by the subgroup generated by $\kappa_{2}$ and $\kappa_{3}$. A direct computation of the eigenvalues and eigenvectors shows that the closed trajectory and the equilibria form a network where the two-dimensional unstable (resp. stable) manifold of $\mathbf{C}$ coincides with the two-dimensional stable (resp.unstable) manifold of $\pm \mathbf{v}$ (resp. $\pm \mathbf{w}$ ). 
Since all the heteroclinic connections are contained in fixed point subspaces, there is no swicthing. Moreover, the network is asymptotically stable by the criteria of Krupa and Melbourne [17], [18].

For $\xi>0$ the $\mathbf{S O}(2)$ symmetry is broken and so are the twodimensional connections. The only symmetries remaining are $\gamma_{1}=\kappa_{\pi}$ and $\gamma_{2}=\kappa_{2} \kappa_{3}$. The symmetry-breaking term $\left(0,0, h_{1}(x), h_{2}(x)\right)$ is tangent to the invariant sphere so it is still flow invariant and properties (P1) and (P2) hold. The perturbation mantain the flow-invariance of the lines that were fixed by $\kappa_{2}$ and $\kappa_{3}$, and the equilibria and the periodic trajectory are preserved together with their stability, and properties (P3-P4) hold. Using Melnikov's Method, Aguiar [1] proved that the two-dimensional manifolds of the periodic trajectory and of the saddle-foci intersect transversely. Hence property (P5) holds.

\section{Local Dynamics neAR the SADDles}

Here and in the next two sections, we define the setup for the proof of swicthing near the network. This section contains mostly notation and coordinates used in the rest of the paper.

We restrict our study to $\mathbf{S}^{3}$ since this is a compact and flow-invariant manifold that captures all the dynamics. When we refer to the stable/unstable manifold of an invariant saddle, we mean the local stable/unstable manifold of that saddle.

We use Samovol's theorem to linearize the flow around each saddle - equilibrium or closed trajectory. We then introduce local cylindrical coordinates and define a neighbourhood with boundary transverse to the linearised flow. For each saddle, we obtain the expression of the local map that sends points in the boundary where the flow goes in, into points in the boundary where it goes out. These expressions will be used in the sequel to obtain a geometrical description of the discretised flow.

5.1. Coordinates near equilibria. Let $\mathbf{v}$ and $\mathbf{w}$ stand for any of the two symmetry-related equilibria $\pm \mathbf{v}$ and $\pm \mathbf{w}$, respectively. By Samovol's theorem [22] $f$ may be linearized around them, since nonresonance is automatic here. In cylindrical coordinates $(\rho, \theta, z)$ the linearizations take the forms:

$$
\left\{\begin{array} { l } 
{ \dot { \rho } = - C _ { v } \rho } \\
{ \dot { \theta } = 1 } \\
{ \dot { z } = E _ { v } z }
\end{array} \quad \left\{\begin{array}{l}
\dot{\rho}=E_{w} \rho \\
\dot{\theta}=1 \\
\dot{z}=-C_{w} z .
\end{array}\right.\right.
$$

We consider cylindrical neighbourhoods of $\mathbf{v}$ and $\mathbf{w}$ in $\mathbf{S}^{3}$ of radius $\varepsilon>0$ and height $2 \varepsilon$. Their boundaries consist of three components (see figure 3):

- The cylinder wall parametrized by $x \in \mathbf{R}(\bmod 2 \pi)$ and $|y| \leq \varepsilon$ with the usual cover $(x, y) \mapsto(\varepsilon, x, y)=(\rho, \theta, z)$. 


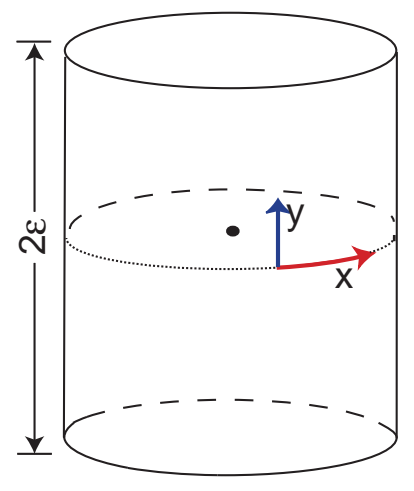

(a)

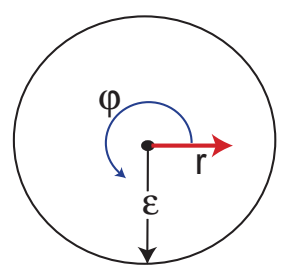

(b)

Figure 3. Coordinates on the boundaries of the neighbourhood of $\mathbf{v}$ and $\mathbf{w}$ : (a) cylinder wall (b) top and bottom.

- Two disks, the top and the bottom of the cylinder. We take polar coverings of these disks: $(r, \varphi) \mapsto(r, \varphi, j \varepsilon)=(\rho, \theta, z)$ where $j \in\{-,+\}, 0 \leq r \leq \varepsilon$ and $\varphi \in \mathbf{R}(\bmod 2 \pi)$.

change We use $x$ for the angular coordinate on the cylinder wall so as to avoid confusion with the angular coordinate on the disks when dealing with the local maps.

Note that the two flows defined by (2) have symmetry $\mathbf{Z}_{2} \oplus \mathbf{S O}(2)$ given by $z \mapsto-z$ and rotation around the $z$ axis. This is an artifact of the linearisation and has nothing to do with the original symmetries, but it will be useful in simplifying statements in the next sections.

5.2. Local dynamics near $\mathbf{v}$. The cylinder wall is denoted $H_{v}^{i n}$. Trajectories starting at interior points of $H_{v}^{i n}$ go inside the cylinder in positive time and $H_{v}^{i n} \cap W^{s}(\mathbf{v})$ is parametrized by $y=0$. The set of points in $H_{v}^{i n}$ with positive (resp. negative) second coordinate is denoted $H_{v}^{i n,+}$ (resp. $H_{v}^{i n,-}$ ).

The top and the bottom of the cylinder are denoted $H_{v}^{\text {out, }+}$ and

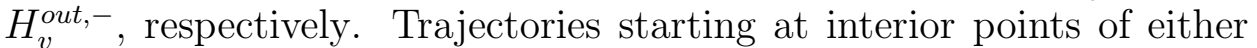
$H_{v}^{\text {out },+}$ or $H_{v}^{\text {out,--}}$ go inside the cylinder in negative time (see figure 4).

After linearization $W^{u}(\mathbf{v})$ is the z-axis, intersecting $H_{v}^{\text {out },+}$ at the origin of coordinates of $H_{v}^{\text {out },+}$. Trajectories starting at $H_{v}^{i n, j}, j \in$ $\{+,-\}$ leave the cylindrical neighbourhood at $H_{v}^{o u t, j}$. The orientation of the $z$-axis may be chosen to have $[\mathbf{v} \rightarrow j \mathbf{w}]$ meeting $H_{v}^{\text {out }, j}$.

The local map near $\mathbf{v}, \phi_{v}: H_{v}^{i n,+} \rightarrow H_{v}^{\text {out },+}$ is given by

$$
\phi_{v}(x, y)=\left(K_{v} y^{\delta_{v}},-\frac{1}{E_{v}} \ln y+x+\frac{1}{E_{v}} \ln (\varepsilon)\right)=(r, \phi),
$$



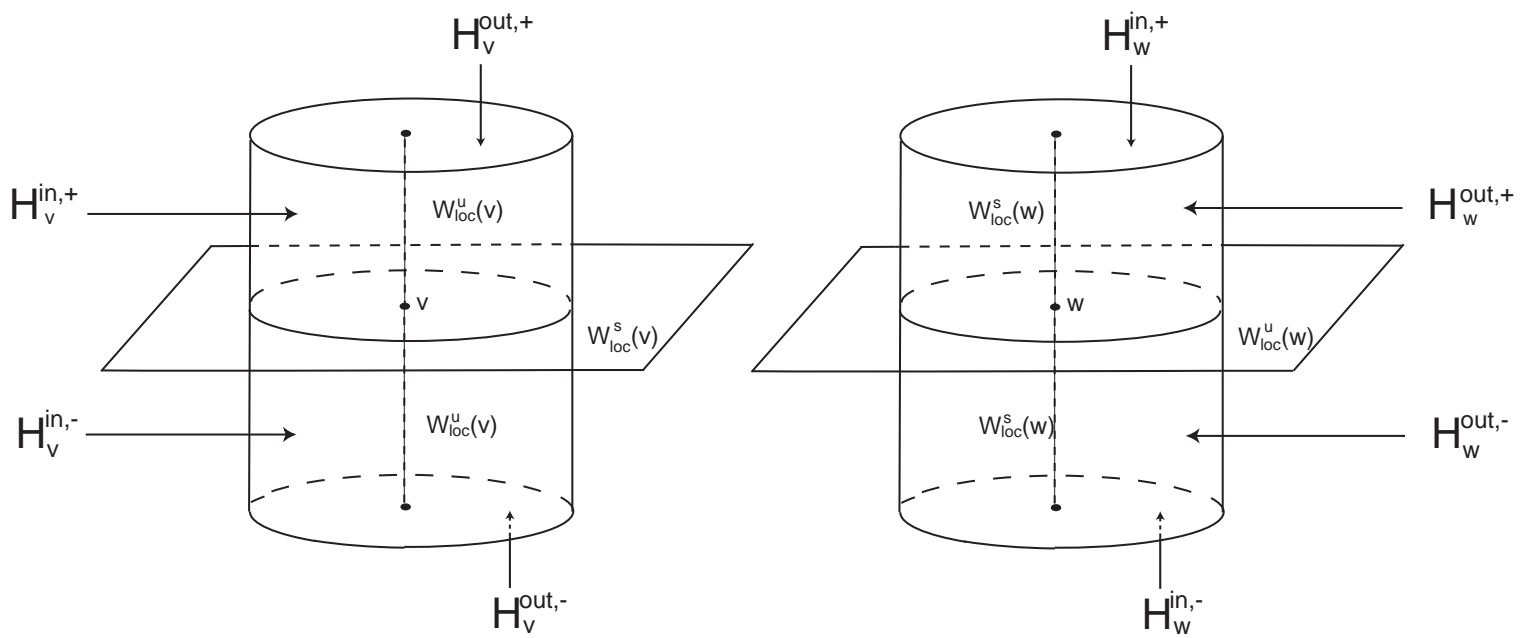

Figure 4. Neighbourhoods of the saddle-foci. Left: once the flow enters the cylinder transversely across the wall $H_{v}^{i n} \backslash W_{l o c}^{s}(\mathbf{v})$ it leaves it transversely across the cylinder top $H_{v}^{\text {out },+}$ and bottom $H_{v}^{\text {out,-}}$. Right: the flow enters the cylinder transversely across top $H_{w}^{i n,+} \backslash W_{l o c}^{s}(\mathbf{w})$ and bottom $H_{w}^{i n,-} \backslash W_{l o c}^{s}(\mathbf{w})$ and leaves it transversely across the wall $H_{w}^{\text {out }}$. Inside the two cylinders the vector field is linear.

where

$$
\delta_{v}=\frac{C_{v}}{E_{v}}>0, \quad K_{v}=\varepsilon^{1-\delta_{v}}>0 \quad \text { and } \frac{1}{E_{v}}>0
$$

The expression for the local map from $H_{v}^{\text {in,-- }}$ to $H_{v}^{\text {out,- }}$ is the same.

5.3. Local dynamics near w. After linearization, $W^{s}(\mathbf{w})$ is the $z$ axis, intersecting the top and bottom of the cylinder at the origin of the coordinates. We denote by $H_{w}^{i n, j}, j \in\{-,+\}$, the component that $[j \mathbf{v} \rightarrow \mathbf{w}] \cap H_{w}^{i n, j} \neq \emptyset$. Trajectories starting at interior points of $H_{w}^{i n, \pm}$ go inside the cylinder in positive time (see figure 4 ).

Trajectories starting at interior points of the cylinder wall $H_{w}^{\text {out }}$ go inside the cylinder in negative time. The set of points in $H_{w}^{\text {out }}$ whose second coordinate is positive (resp. negative) is denoted $H_{w}^{\text {out },+}$ (resp. $\left.H_{w}^{\text {out },-}\right)$ and $H_{w}^{\text {out }} \cap W^{u}(\mathbf{w})$ is parametrized by $y=0$. The orientation of the $z$-axis may be chosen to have trajectories that start at $H_{w}^{i n, j} \backslash W^{s}(\mathbf{w}), j \in\{+,-\}$ leaving the cylindrical neighbourhood at 
$H_{w}^{\text {out }, j}$. The local map near $\mathbf{w}, \phi_{w}: H_{w}^{i n,+} \backslash W^{s}(\mathbf{w}) \rightarrow H_{w}^{o u t,+}$ is

$$
\phi_{w}(r, \varphi)=\left(\frac{1}{E_{w}} \ln (\varepsilon)-\frac{1}{E_{w}} \ln r+\varphi, K_{w} r^{\delta_{w}}\right)=(x, y)
$$

where

$$
\delta_{w}=\frac{C_{w}}{E_{w}}>0, \quad K_{w}=\varepsilon^{1-\delta_{w}}>0 \quad \text { and } \quad \frac{1}{E_{w}}>0 .
$$

The same expression holds for the local map from $H_{w}^{i n,-} \backslash W^{s}(\mathbf{w})$ to $H_{w}^{o u t,-}$.

5.4. Local dynamics near the closed trajectory C. Consider a local cross section $S$ to the flow at $p \in \mathbf{C}$. The Poincaré first return map defined on $S$ may be linearized around the hyperbolic fixed point $p$ using Samovol's Theorem. Suspending the linear map yields, in cylindrical coordinates, the differential equations:

$$
\left\{\begin{array}{l}
\dot{\rho}=-C_{C}(\rho-1) \\
\dot{\theta}=1 \\
\dot{z}=E_{C} z
\end{array}\right.
$$

that are locally orbitally equivalent to the original flow. In these coordinates, $\mathbf{C}$ corresponds to the circle $\rho=1$ and $z=0, W^{s}(\mathbf{C})$ is the plane $z=0$ and $W^{u}(\mathbf{C})$ is the cylinder $\rho=1$.

We work with a hollow three-dimensional cylindrical neighbourhood of $\mathbf{C}$ with boundary $H_{C}^{i n} \cup H_{C}^{\text {out }}$, where trajectories starting in $H_{C}^{\text {in }}$ (resp. $H_{C}^{\text {out }}$ ) go into the neighbourhood in positive (resp. negative) small time. In what follows we establish some notation for components of the boundary (see figure 5).

The components of $H_{C}^{i n}$ are the two cylinder walls, $H_{C,+}^{i n}$ and $H_{C,-}^{i n}$ locally separated by $W^{u}(\mathbf{C})$ and parametrized by the covering map:

$$
(x, y) \mapsto(1 \pm \varepsilon, x, y)=(\rho, \theta, z),
$$

where $x \in \mathbf{R}(\bmod 2 \pi),|y|<\varepsilon$. We denote by $H_{C,+}^{i n}$ the component with $\rho=1+\varepsilon$.

In these coordinates, $H_{C}^{i n} \cap W^{s}(\mathbf{C})$ is the union of the two circles $y=0$ in the two components. It divides $H_{C,+}^{i n}$ in two parts, $H_{C,+}^{i n,+}$ and $H_{C,+}^{i n,-}$, parametrized, respectively, by positive and negative $y$, with a similar convention for $H_{C,-}^{i n,+}$ and $H_{C,-}^{i n,-}$.

The components $H_{C}^{\text {out },+}$ and $H_{C}^{\text {out },-}$ of $H_{C}^{\text {out }}$ are two anuli, locally separated by $W^{s}(\mathbf{C})$ and parametrized by the covering:

$$
(r, \varphi) \mapsto(r, \varphi, \pm \varepsilon)=(\rho, \theta, z),
$$

for $1-\varepsilon<r<1+\varepsilon$ and $\varphi \in \mathbf{R}(\bmod 2 \pi)$ and where $H_{C}^{o u t,+}$ is the component corresponding to the $+\operatorname{sign}$ and $H_{C}^{\text {out }} \cap W^{u}(\mathbf{C})$ is the union of two circles parametrized by $r=1$. 


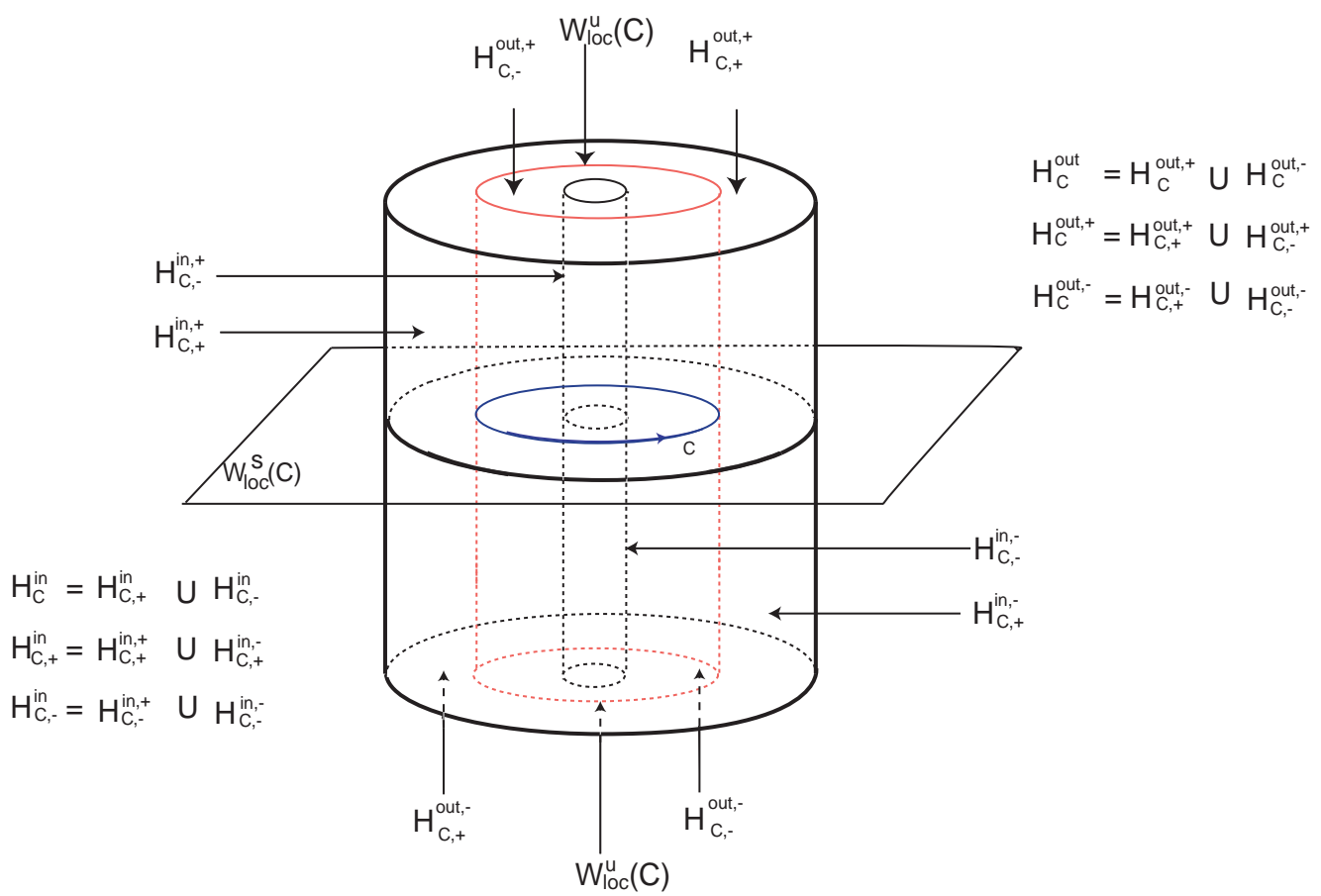

Figure 5. Neighbourhood of the closed trajectory C. The flow enters the hollow cylinder transversely across cylinder walls $H_{C, \pm}^{i n}$ and leaves it transversely across top $H_{C,+}^{o u t}$ and bottom $H_{C,-}^{o u t}$.

Denote by $H_{C,+}^{\text {out }, k}\left(\operatorname{resp} . H_{C,-}^{\text {out }, k}\right), k \in\{+,-\}$ the set parametrized by $1<r<1+\varepsilon$ (resp. $1-\varepsilon<r<1$ ) in $H_{C}^{\text {out }, k}$. In these coordinates the local map $\phi_{C}: H_{C, j}^{i n, k} \rightarrow H_{C, j}^{\text {out }, k}, j, k \in\{+,-\}$, is given by

$$
\phi_{C}(x, y)=\left(j K_{c} y^{\delta_{c}}+1, \frac{1}{E_{c}} \ln (\varepsilon)-\frac{1}{E_{c}} \ln y+x\right)=(r, \varphi),
$$

where

$$
\delta_{c}=\frac{C_{c}}{E_{c}}>0 \quad K_{c}=\varepsilon^{1-\delta_{C}}>0 \text { and } \frac{1}{E_{c}}>0 .
$$

\section{Geometry NeAR the SADDLES}

The coordinates and notation of section 3 may now be used to analyse the geometry of the local dynamics near each saddle. The manifold $W^{s}(\mathbf{v})$ separates the cylindrical neighbourhood of $\mathbf{v}$ into an upper and a lower component, mapped into neighbourhoods of $P w$ and $-\mathbf{w}$, respectively. We show here that initial conditions lying on a segment on the upper part of the cylindrical wall around $\mathbf{v}$ and ending at $W^{s}(\mathbf{v})$ 
are mapped into points on a spiral on the top of the cylinder $H_{v}^{\text {out }}$ where the flow goes out (figure 6).

Initial conditions on a spiral on the top of cylindrical neighbourhood of $\mathbf{w}$ are then shown to be mapped into points on a helix around the cylinder accumulating on $W^{u}(\mathbf{w})$ (figure 6). Since $W^{s}(\mathbf{C})$ is transverse to $W^{u}(\mathbf{w})$, then the helix on $H_{w}^{\text {out },+}$ is mapped across $W^{s}(\mathbf{C})$ on $H_{C}^{\text {in }}$ infinitely many times. This will be used in section 7 to obtain, in any segment on $H_{v}^{i n,+}$, infinitely many intervals that are mapped into segments on $H_{C}^{i n}$ ending at $W^{s}(\mathbf{C})$.

Then the image of a segment ending at $W^{s}(\mathbf{C})$ on one of the walls of $H_{C}^{i n}$ is shown to be mapped into a curve accumulating on $W^{u}(\mathbf{C}) \cap$ $H_{C}^{\text {out }}$ (see figure 7 ). This curve meets $W^{s}(\mathbf{v})$ infinitely many times by transversality and it is thus mapped across $W^{s}(\mathbf{v})$ on $H_{v}^{\text {in }}$ infinitely many times. Again, we will use this in section 7 to obtain an infinitely many intervals that are mapped into segments on $H_{v}^{i n}$ ending at $W^{s}(\mathbf{v})$.

This structure of segments containing intervals that are successively mapped into segments will allow us to establish a recurrence in section 8 and to construct nested sequences of intervals containing the initial conditions for switching.

Definition 1. $A$ segment $\beta$ on $H_{v}^{\text {in }}$ (resp.: $\left.H_{C}^{i n}\right)$ is a smooth regular parametrized curve $\beta:[0,1] \rightarrow H_{p}^{i n}\left(\right.$ resp.: $\left.\beta:[0,1] \rightarrow H_{C}^{i n}\right)$, that meets $W^{s}(\mathbf{v})$ (or $W^{s}(\mathbf{C})$ ) transverselly at the point $\beta(1)$ only and such that, writing $\beta(s)=(x(s), y(s))$, both $x$ and $y$ are monotonic functions of $s$.

The coordinates $(x, y)$ may be chosen so as to make the angular coordinate $x$ an increasing or decreasing function of $s$ as convenient.

Definition 2. Let $U$ be an open set in a plane in $\mathbf{R}^{n}$ and $p \in U$. A spiral on $U$ around $p$ is a curve $\alpha:[0,1) \rightarrow U$ satisfying $\lim _{s \rightarrow 1^{-}} \alpha(s)=$ $p$ and such that, if $\alpha(s)=\left(\alpha_{1}(s), \alpha_{2}(s)\right)$ are its expressions in polar coordinates $(\rho, \theta)$ around $p$, then $\alpha_{1}$ and $\alpha_{2}$ are monotonic, with

$$
\lim _{s \rightarrow 1^{-}}\left|\alpha_{2}(s)\right|=+\infty \text {. }
$$

It follows that $\alpha_{1}$ is a decreasing function of $s$.

Proposition 1. A segment $\beta$ on $H_{v}^{i n,+}$ (resp. $\left.H_{v}^{i n,-}\right)$ is mapped by $\phi_{v}$ into a spiral on $H_{v}^{\text {out, }+}$ (resp. $H_{v}^{\text {out, }-}$ ) around $W^{u}(\mathbf{v})$.

Proof. Write $\beta(s)=(x(s), y(s))$ on $H_{v}^{i n,+}$ with $y(s) \geq 0$ monotonically decreasing and choose a parametrization of $H_{v}^{i n,+}$ such that $x(s)$ is monotonically increasing. Then, writing $\phi_{v}(\beta(s))=(r(s), \theta(s))$, it follows from the expression of $\phi_{v}$ in section 5.2 that $r(s)$ is monotonically decreasing while $\theta(s)$ is monotonically increasing. From $\lim _{s \rightarrow 1^{-}} y(s)=$ 0 and $\lim _{s \rightarrow 1^{-}} x(s)=x(1)$ the required limits $\lim _{s \rightarrow 1^{-}} r(s)=0$ and $\lim _{s \rightarrow 1^{-}} \theta(s)=+\infty$ follow. 

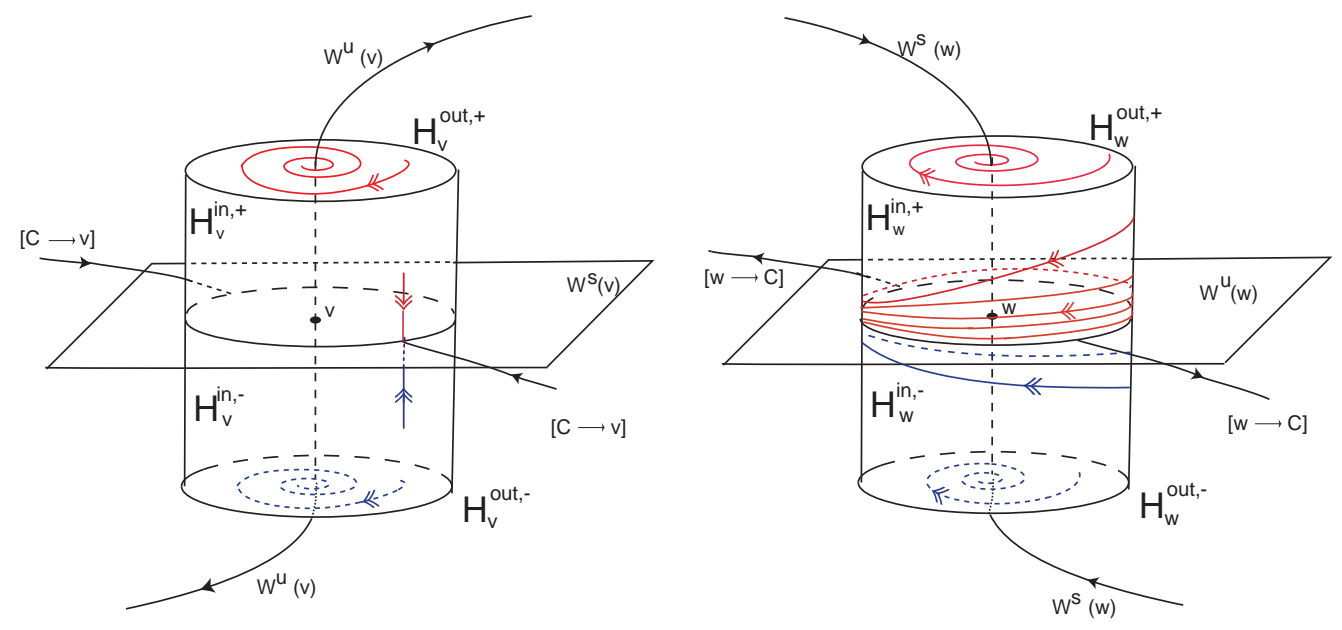

Figure 6. Local Dynamics near the saddle-foci. Left: Near $\mathbf{v}$, any segment on the cylinder wall is mapped into a spiral on the top or bottom of the cylinder. Right: A spiral on the top or bottom of the cylinder near $\mathbf{w}$ is mapped into a helix on the cylinder wall accumulating on $W^{u}(\mathbf{w})$. The double arrows on the segment, spiral and helix indicate correspondence of orientation and not the flow.

Definition 3. Let $a, b \in \mathbf{R}$ such that $a<b$ and let $H$ be a surface parametrized by a cover $(\theta, h) \in \mathbf{R} \times[a, b]$ where $\theta$ is periodic. $A$ helix on $H$ accumulating on the circle $h=h_{0}$ is a curve $\gamma:[0,1) \rightarrow H$ such that its coordinates $(\theta(s), h(s))$ are monotonic functions of $s$ with

$$
\lim _{s \rightarrow 1^{-}} h(s)=h_{0} \quad \text { and } \quad \lim _{s \rightarrow 1^{-}}|\theta(s)|=+\infty .
$$

Proposition 2. A spiral on $H_{w}^{i n,+}$ (resp. $H_{w}^{i n,-}$ ) around $W^{s}(\mathbf{w})$ is mapped by $\phi_{w}$ into a helix on $H_{w}^{\text {out },+}$ (resp. $H_{w}^{\text {out },-}$ ) accumulating on the circle $H_{w}^{\text {out }} \cap W^{u}(\mathbf{w})$.

Proof. Parametrize $H_{w}^{i n,+}$ so that a spiral $\sigma(s)=(r(s), \theta(s))$ around $W^{s}(\mathbf{w})$ has $\theta(s)$ increasing with $s$. The expression of $\phi_{w}$ of section 5.3 ensures that in $\phi_{w}(\sigma(s))=(x(s), y(s))$ we have $y$ decreasing with $s$ and $x$ increasing with $s$. The limits in the definiton of helix follow from the form of $\phi_{w}$ and from $\lim _{s \rightarrow 1^{-}} r(s)=0$ and $\lim _{s \rightarrow 1^{-}} \theta(s)=+\infty$.

Proposition 3. A segment $\beta$ on $H_{C,+}^{i n,+}$ is mapped by $\phi_{C}$ into a helix on $H_{C,+}^{\text {out },+}$ accumulating on the circle $W^{u}(\mathbf{C}) \cap H_{C}^{\text {out },+}$.

The proof, as in Propositions 1 and 2, consists of using the expression of $\phi_{C}$ of section 5.4 after a suitable choice of orientation in $H_{C,+}^{i n,+}$. Using the symmetries of the linearised flow, it follows that Proposition 3 also 


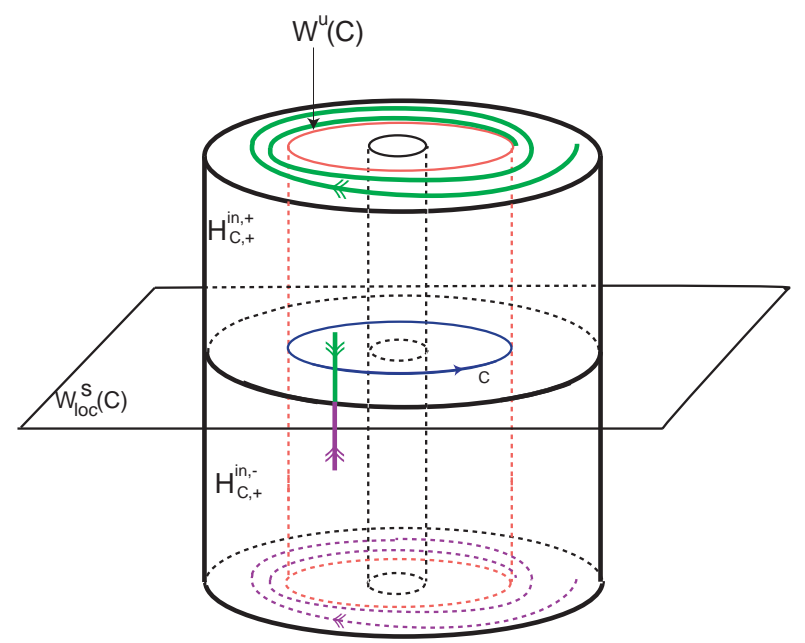

Figure 7. Local dynamics near the closed trajectory $\mathbf{C}$. A segment on the wall ending at $W^{s}(\mathbf{C})$ is mapped into a curve accumulating on $W^{u}(\mathbf{C}) \cap H_{C}^{\text {out }}$.

holds for a segment $\beta$ on $H_{C-}^{\text {in,+ }}$ and for $H_{C-}^{\text {out },+}$, as well as for a segment $\beta$ on $H_{C+}^{i n,-}$ and for $H_{C+}^{\text {out,-}}$ and for $\beta$ on $H_{C-}^{\text {in,- }}$ and for $H_{C-}^{\text {out,-}}$, considering the circle $W^{u}(\mathbf{C}) \cap H_{C}^{\text {out,- }}$ (see figure 7 ).

\section{First RETURN TO $\mathbf{v}$}

Let $p$ and $q$ be two nodes of $\Sigma$ such that there is a connection $[p \rightarrow q]$. The transition map $\Psi_{p, q}$ from $H_{p}^{\text {out }}$ to $H_{q}^{\text {in }}$ follows the trajectory $[p \rightarrow q]$ in flow-box fashion. In this section we use this information to put together the local behaviour of trajectories that start near $\pm \mathbf{v}$. To simplify the reading, we omit the - and + signs.

Let $P \in H_{w}^{\text {out }}$ be one of the points where $[w \rightarrow \mathbf{C}]$ meets $H_{w}^{\text {out }}$. For small $a, b>0$, the rectangle $\left[-\frac{a}{2}, \frac{a}{2}\right] \times\left[-\frac{b}{2}, \frac{b}{2}\right]$ is mapped diffeomorphically into $H_{w}^{\text {out }}$ by the parametrization that maps the origin to $P$ (figure 8). Its image, that we denote by $R_{w}$, will be called a rectangle in $H_{w}^{\text {out }}$ centered at $P$ with height $b$.

The vertical sides of $R_{w}$ are the images of the segments $\left( \pm \frac{a}{2}, y\right)$ with $y \in\left[-\frac{b}{2}, \frac{b}{2}\right]$. Rectangles in $H_{C}^{\text {out }}$ centered at a given point are defined in the same way; we denote them by $R_{C}$.

By Propositions 1 and 2, the map

$$
\eta=\phi_{w} \circ \Psi_{v, w} \circ \phi_{v}: H_{v}^{i n} \rightarrow H_{w}^{o u t}
$$

maps a segment $\beta$ on $H_{v}^{\text {in }}$ infinitely many times across any small rectangle in $H_{w}^{\text {out }}$ centered at a point in $W^{u}(\mathbf{w})$. 


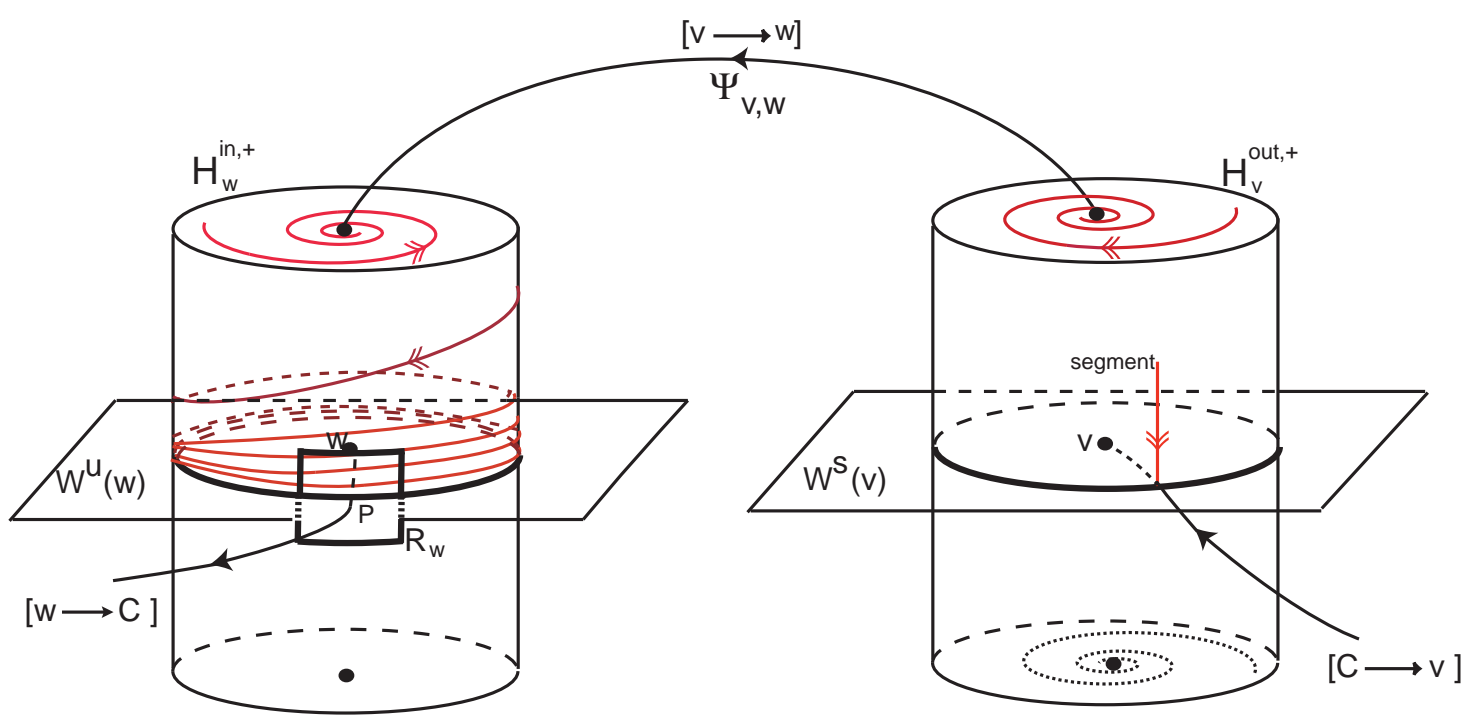

FiguRE 8. Transition from $\mathbf{v}$ to $\mathbf{w}$ : a segment on $H_{v}^{i n}$ is mapped into spirals on $H_{v}^{\text {out }}$ around $W^{u}(\mathbf{v}) \cap H_{v}^{\text {out }}$ and $W^{s}(\mathbf{w}) \cap H_{w}^{i n}$. The spiral is then mapped into a helix on $H_{w}^{\text {out }}$ accumulating on $W^{u}(\mathbf{w})$ and crossing infinitely many times a rectangle $R_{w}$ centered at one of the connections starting at $\mathbf{w}$ (see also figure 9 ). Double arrows indicate orientation of the segment and not the flow.

An admissible family of intervals $\mathcal{I}=\left\{\left[a_{i}, b_{i}\right]\right\}_{i \in \mathbf{N}}$ is one that satisfies

$$
0<a_{i}<b_{i}<a_{i+1}<1 \quad \text { and } \quad \lim _{i \rightarrow \infty} a_{i}=1 .
$$

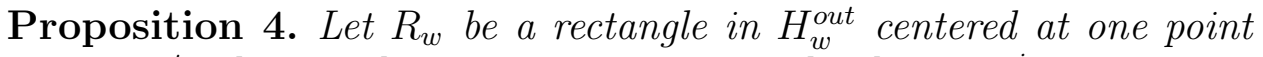
$P$ of $H_{w}^{\text {out }} \cap[\mathbf{w} \rightarrow \mathbf{C}]$. For any segment $\beta:[0,1] \longrightarrow H_{v}^{\text {in }}$ there is an admissible family of intervals $\left\{\left[\sigma_{i}, \rho_{i}\right]\right\}_{i \in \mathbf{N}}$ such that:

(1) each closed interval $\left[\sigma_{i}, \rho_{i}\right]$ satisfies $\eta \circ \beta\left(\left[\sigma_{i}, \rho_{i}\right]\right) \subset R_{w}$;

(2) each open interval $\left(\rho_{i+1}, \sigma_{i}\right)$ satisfies $\eta \circ \beta\left(\left(\rho_{i+1}, \sigma_{i}\right)\right) \cap R_{w}=\emptyset$;

(3) the family of curves $\left\{\eta \circ \beta\left(\left[\sigma_{i}, \rho_{i}\right]\right)\right\}$ accumulates uniformly on $W^{u}(\mathbf{w}) \cap R_{w}$ as $i \rightarrow+\infty$.

This also holds for the local map around $\mathbf{C}$, with $\mathbf{C}, \phi_{C}$ and $\mathbf{C}$ where we have written $\mathbf{w}, \eta$ and $\mathbf{v}$.

Proof. Writing $(x(s), y(s))$ for the coordinates of the helix $\eta \circ \beta(s)$ on the cylinder wall, we have that $y$ decreases with $s$ and that $x(s)$ can be taken as an increasing function of $s$ by choosing compatible orientations in $H_{v}^{i n}$ and $H_{w}^{o u t}$ and, if necessary, by restricting the domain of $\beta$ to a smaller interval $\left(s_{1}, 1\right)$. In particular the helix $\eta \circ \beta$ may be seen as 
a graph $(x, y(x))$ where $x \in[x(0),+\infty)$ and where $y$ is a decreasing function of $x$ with $\lim _{x \rightarrow \infty} y(x)=0$ (figure 9 ).

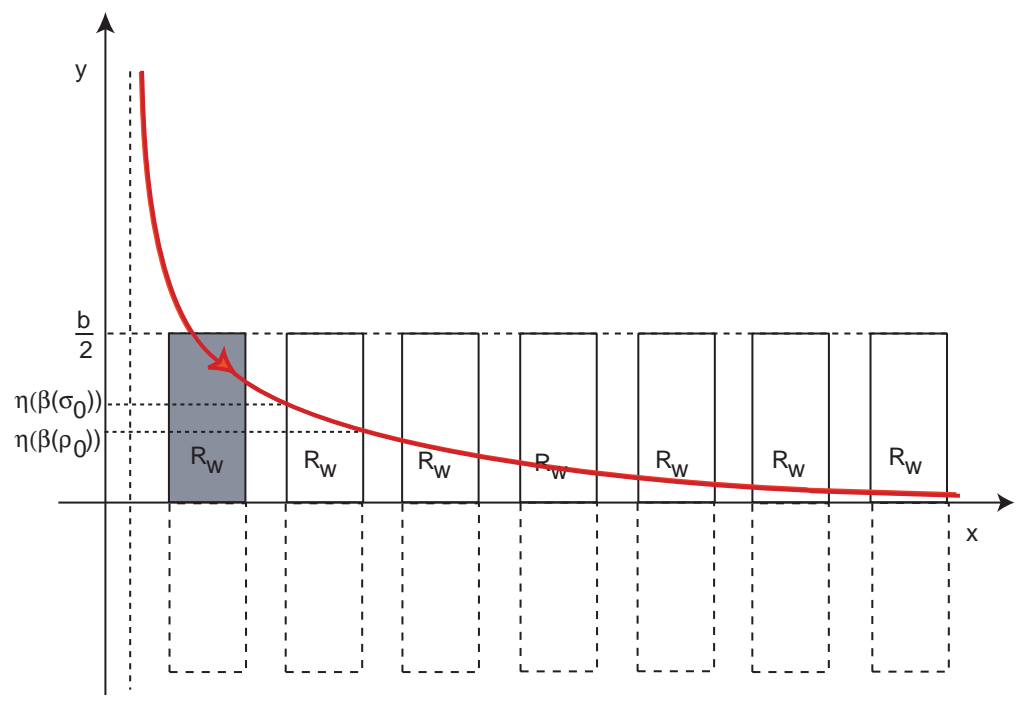

Figure 9. A helix on a periodic cover of the cylinder wall $H_{w}^{\text {out }}$. After it meets the first (shaded) copy of the rectangle, the helix will intersect the rectangle at intervals whose image accumulates on the curve $W^{u}(\mathbf{w})$, represented here by the $x$-axis.

On $H_{w}^{\text {out }}$ the rectangle $R_{w}$ is $[n-a / 2, n+a / 2] \times[-b / 2, b / 2]$ with $n \in \mathbf{N}$. Let $\sigma_{0}$ be the smallest value of $s \in\left(s_{1}, 1\right)$ such that $(x(s), y(s))$ lies on the left vertical side of $R_{w}$, with $y\left(\sigma_{0}\right)<\frac{b}{2}$ as in figure 9 . Then $y(s)<\frac{b}{2}$ for all $s \in\left[\sigma_{0}, 1\right)$.

The sequences defining the family of intervals are obtained from points where the helix meets successive copies of the vertical sides of $R_{w}$ with $x\left(\sigma_{i}\right)=n_{0}+i-a / 2$ and $x\left(\rho_{i}\right)=n_{0}+i+a / 2$. The proof for $\phi_{C}$ is similar.

Proposition 5. Given a segment $\beta:[0,1] \longrightarrow H_{v}^{i n}$, a rectangle $R_{w}$ of sufficiently small height and the family of intervals $\left\{\left[\sigma_{i}, \rho_{i}\right]\right\}_{i \in \mathbf{N}}$ of Proposition 4. Then for sufficiently large $i$ there are $\tau_{i}$ with $\sigma_{i}<\tau_{i}<\rho_{i}$ such that $\Psi_{w, C} \circ \eta\left(\beta\left(\tau_{i}\right)\right) \in W^{s}(\mathbf{C})$ and $\Psi_{w, C} \circ \eta \circ \beta$ maps each one of the intervals $\left[\sigma_{i}, \tau_{i}\right]$ and $\left[\tau_{i}, \rho_{i}\right]$ into a segment on one of the sets $H_{C}^{i n,+}$ and $H_{C}^{i n,-}$. This also holds for $\Psi_{C, v} \circ \phi_{C}: H_{C}^{i n} \longrightarrow H_{v}^{i n, \pm}$ with the appropriate changes.

Proof. Since $W^{s}(\mathbf{C}) \cap H_{w}^{\text {out }}$ meets $W^{u}(\mathbf{w}) \cap H_{w}^{\text {out }}$ transverselly (property $(\mathrm{P} 5))$ then if the height of $R_{w}$ is small, $W^{s}(\mathbf{C})$ does not meet its vertical sides. Each one of the images $\eta \circ \beta\left(\left[\sigma_{i}, \rho_{i}\right]\right)$ meets $W^{s}(\mathbf{C}) \cap H_{w}^{\text {out }}$ 
transversely at a single point $\eta \circ \beta\left(\tau_{i}\right)$, since they accumulate uniformly on $W^{u}(\mathbf{w}) \cap R_{w}$ as $i \rightarrow+\infty$. The monotonicity of the coordinates of $\eta \circ \beta$ will be preserved by $\Psi_{w, C}$ close to $W^{u}(\mathbf{w})$. Each component of $\eta \circ \beta\left(\left[\sigma_{i}, \rho_{i}\right]\right) \backslash\left\{\eta \circ \beta\left(\tau_{i}\right)\right\}$ will be mapped into a segment, one into each connected component of $H_{C}^{i n, j} \backslash W^{s}(\mathbf{C})$. The proof for $\Psi_{C, v} \circ \phi_{C}$ is analogous.

\section{Switching NeAR the Heteroclinic Network}

In this section, we put together the information about the first return map to $H_{v}^{i n}$. In sections 6 and 7 we have found that a segment ending at a stable manifold contains intervals that are mapped into segments ending at the stable manifold of the next node. Starting with a segment on in $H_{v}^{i n}$, here this is used recursively to obtain sequences of nested intervals containing initial conditions that follow sequences heteroclinic connections.

We say that the path $s^{k}=\left(c_{j}\right)_{j \in\{1, \ldots, k\}}$ of order $k$ on the network $\Sigma$ is inside the path $t^{k+l}=\left(d_{j}\right)_{j \in\{1, \ldots, k+l\}}$ of order $k+l$ (denoted $s^{k} \prec t^{k+l}$ ) if $c_{j}=d_{j}$ for all $j \in\{1, \ldots, k\}$.

The family of closed intervals $\mathcal{I}=\left\{I_{i}\right\}_{i \in \mathbf{N}}$ is inside the family $\tilde{\mathcal{I}}=$ $\left\{\tilde{I}_{i}\right\}_{i \in \mathbf{N}}(\mathcal{I}<\tilde{\mathcal{I}})$ if, for all $i \in \mathbf{N}, I_{i} \subset \tilde{I}_{i}$. If $\tilde{\mathcal{I}}$ is admissible in the sense of section 7 and $\mathcal{I}<\tilde{\mathcal{I}}$ then $\mathcal{I}$ is also admissible, provided none of its intervals consists of a point.

Theorem 6. There is finite switching near the network $\Sigma$ defined by a vector field satisfying (P1)-(P5).

Proof. Given a path, we want to find trajectories that follow it into the neighbourhoods of section 5 going through small disks in $H_{v}^{\text {out }}$ around $W^{u}( \pm \mathbf{v})$ and through rectangles in $H_{w}^{\text {out }}$ and $H_{C}^{\text {out }}$ centered at the connections. Without loss of generality we only consider paths $s^{k}=\left(c_{j}\right)_{j \in\{1, \ldots, k\}}$ starting with $c_{1}=[ \pm \mathbf{v} \rightarrow \pm \mathbf{w}]$.

Take a segment $\beta$ on $H_{v}^{i n, \pm}$ of points that follow the first connection $c_{1}$. We will construct admissible families of intervals $\mathcal{I}\left(c_{1}, \ldots, c_{n}\right)=$ $\left\{I_{i}\right\}_{i \in \mathbf{N}}$ recursively, such that points in $\beta\left(I_{i}\right)$ follow $\left(c_{1}, \ldots, c_{n}\right)$ and the image of $\beta\left(I_{i}\right)$ by the transition maps is a segment. We will show that $s^{k} \prec s^{k+l}$ implies $\mathcal{I}\left(s^{k}\right)>\mathcal{I}\left(s^{k+l}\right)$ and thus the process will be recursive.

By Propositions 4 and 5, there is an admissible family of intervals $\mathcal{I}\left(c_{1}, c_{2}\right)=\left\{I_{i}\right\}_{i \in \mathbf{N}}$ such that $\beta\left(I_{i}\right)$ is mapped by $\Psi_{w, C} \circ \phi_{w} \circ \Psi_{v, w} \circ$ $\phi_{v}$ into a segment on $H_{C}^{i n, \pm}$ with the choice of sign appropriate for the next connection $c_{3}$. Applying the second part of Propositions 4 and 5 to this segment, we obtain an admissible family of intervals $\mathcal{I}\left(c_{1}, c_{2}, c_{3}\right)<\mathcal{I}\left(c_{1}, c_{2}\right)$ corresponding to points that follow $\left(c_{1}, c_{2}, c_{3}\right)$ and to intervals that are mapped into a segment on $H_{v}^{i n, \pm}$ with the choice of sign appropriate for following the connection $c_{4}$. 

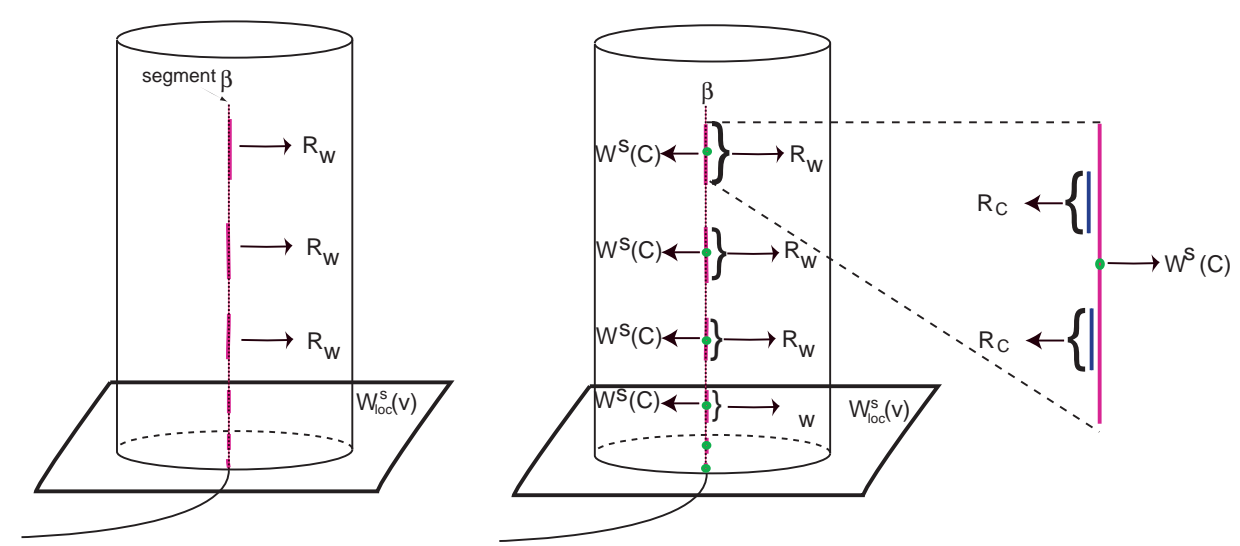

Figure 10. On a segment $\beta$ on $H_{v}^{\text {in }}$, there are infinitely many small segments that are mapped by $\eta$ into $R_{w}$, each one containing a point mapped into $W^{s}(\mathbf{C})$. The small segments contain smaller ones that are mapped into $R_{C}$ and this may be continued, forming a nested sequence.

In Proposition 5 we assume the height of the rectangle $R_{w}$ is small and we reduce it if necessary. This is done to ensure that inside $R_{w}$ the stable manifold $W^{s}(\mathbf{C})$ is the graph of a function and thus a helix only meets it once at each turn. However, as soon as the choice of height is made it may be kept throughout the proof and thus the construction of $\mathcal{I}\left(s^{k}\right)$ is recursive, proving finite switching near $\Sigma$.

Theorem 7. There is infinite switching near the network $\Sigma$ defined by a vector field satisfying (P1)-(P5).

Proof. Fix an infinite path $s^{\infty}=\left(c_{j}\right)_{j \in \mathbf{N}}$ on $\Sigma$. For each $k \in \mathbf{N}$ define the finite path $s^{k}$ of order $k$ by $s^{k}=\left(c_{j}\right)_{j \in\{1, \ldots, k\}}$, with $s^{k} \prec s^{k+1}$. From the proof of Theorem 6 , for each $k$ we have an admissible family of intervals $\mathcal{I}\left(s^{k}\right)=\left\{J_{k i}\right\}_{i \in \mathbf{N}}$ such that $\mathcal{I}\left(s^{k}\right)>\mathcal{I}\left(s^{k+1}\right)$ and all the points in $\beta\left(J_{k i}\right)$ follow $s^{k}$.

Since we have $\mathcal{I}\left(s^{k}\right)>\mathcal{I}\left(s^{k+1}\right)$ then $\Lambda=\bigcap_{k=1}^{\infty} \mathcal{I}\left(s^{k}\right)$ is non-empty because each set $\Lambda_{i}=\bigcap_{k=1}^{\infty} J_{k i}$ is non empty. From the definition of admissible family of intervals, if we take $a_{i} \in \Lambda_{i}$ then $\lim _{i \rightarrow \infty} a_{i}=1$. From the construction we have that $\beta\left(a_{i}\right)$ follows $s^{\infty}$. Thus, we have obtained a sequence of points $\beta\left(a_{i}\right)$ that accumulate on $\Sigma$ as $i \rightarrow \infty$ and that follow the infinite path.

\section{Final Remarks AND discussion}

9.1. Generalisation. Not all assumptions of Section 3 are essential to prove switching, although some of them simplify the calculations. For instance, the eigenvalues at $\pm \mathbf{v}$ and $\pm \mathbf{w}$ may have any imaginary 
part, not necessarily 1 as in (P3). The proof also works if at one of the pairs of nodes $\pm \mathbf{v}$ or $\pm \mathbf{w}$ the eigenvalues of $d f$ are real, as long as the eigenvalues at the other pair of nodes are not real - it is enough to have one pair of rotating equilibria. Any finite number of one-dimensional connections $[\mathbf{w} \rightarrow \mathbf{C}]$ and $[\mathbf{C} \rightarrow \mathbf{v}]$ could have been used instead of two for each equilibrium.

The existence of swicthing near a networks may be easily generalised to a heteroclinic network involving rotating nodes such that each heteroclinic connection involving a periodic trajectory is transverse and there are no consecutive non transverse heteroclinic connections on the network.

It is not essential to have $\left(\mathbf{Z}_{2} \oplus \mathbf{Z}_{2}\right)$-equivariance. The symmetry $\gamma_{2}$ is used here to obtain the closed trajectory $\mathbf{C}$ and the role of $\gamma_{1}$ is to guarantee that the one-dimensional connections $[\mathbf{v} \rightarrow \mathbf{w}]$ are robust. Symmetries make the existence of the network natural and ensure persistence. Switching will hold for any network having the nodes and connections prescribed here, as long as the remaining assumptions are satisfied.

Estimates for the transition maps may be refined as in Aguiar et al. [4] to show that near this network there is a suspended horseshoe with transition map described as a full shift over a countable set of symbols. The suspended horseshoe has the same shape as the network. Thus, when the $\mathbf{O}(2)$-symmetry of the example in section 4 is broken we have instant chaos. In particular it can be shown that there are periodic orbits that follow any finite path in the network, but this uses techniques beyond the scope of this paper.

Finite switching is present even when all the local maps are expanding, as in the case $C_{v}<E_{v}, C_{w}<E_{w}, C_{C}<E_{C}$. This is due to the rotation around the nodes and is markedly different from the situation where all eigenvalues are real. In the example of section 4 this corresponds to parameter values where the $\mathbf{O}(2)$-symmetric network is a repeller.

A path on the network can also be shadowed by trajectories in $W^{u}(\mathbf{C})$ (see figure 10) because the local unstable manifold of $\mathbf{C}$ meets $H_{v}^{i n}$ at a segment by the transversality assumption (P5). It also follows that there are infinitely many homoclinic connections involving the periodic trajectory $\mathbf{C}$, although there are no homoclinic trajectories involving the equilibria. The geometry of $W^{u}(\mathbf{C})$ gets extremely complicated as we move away from $\mathbf{C}$, since it will accumulate on the whole network, having $\mathbf{v}, \mathbf{w}$ and $\mathbf{C}$ as limit points. A complete description of the nonwandering set for this type of flows is in preparation.

9.2. Discussion. Generic breaking of the $\gamma_{1}$-symmetry destroys the network, as in [14] and [20], by breaking the connections $[\mathbf{v} \rightarrow \mathbf{w}]$. If the remaining assumptions are still satisfied, a weaker form of switching will 
hold: for small symmetry-breaking terms, it may still be possible to find trajectories that visit neighbourhoods of finite sequences of nodes. This is because the spirals on top of the cylinder around $\mathbf{w}$ of figure 6 will be off-centered and will turn a finite number of times around $W^{s}(\mathbf{w})$. From this we may obtain points whose trajectories follow short finite paths on the network. As $W^{u}(\mathbf{v})$ gets closer to $W^{s}(\mathbf{w})$ (as the system moves closer to symmetry) the paths that can be shadowed get longer.

This is in contrast to the findings of Kirk and Rucklidge [14], who claim that there can be no switching in generic systems close to the symmetric case, so a comparison of the settings and results of the two papers is in order at this point. The first caveat is that the $\mathbf{Z}_{2} \oplus$ $\mathbf{Z}_{2}$ representations are different: in our case $F i x\left(\mathbf{Z}_{2} \oplus \mathbf{Z}_{2}\right)=\{0\}$, and there are two transverse 2-dimensional fixed-point subspaces for the isotropy subgroups, whereas in [14], Fix $\left(\mathbf{Z}_{2} \oplus \mathbf{Z}_{2}\right)$ is a plane with two 1-dimensional fixed-point subspaces for the isotropy subgroups. In their setting, our symmetries correspond to the group generated by a rotation of $\pi$ in $\mathbf{S O}(2)$ and by the product of their $\mathbf{Z}_{2} \oplus \mathbf{Z}_{2}$ generators. Both representations occur in the larger group $\mathbf{Z}_{2} \oplus \mathbf{Z}_{2} \oplus \mathbf{S O}(2)$ used in [20]. Moreover, we are assuming the existence of an invariant 3-sphere (a natural assumption in the symmetric context, see [8] ) and it is not evident that in their context such a sphere will exist.

However, the difference in the results of [14] and ours indicates that vector fields with $\mathbf{Z}_{2} \oplus \mathbf{Z}_{2} \oplus \mathbf{S O}(2)$ symmetry have codimension higher than 3 in the universe of general vector fields. This will mean that in general systems close to symmetry what will be observed may depend on the way symmetries are broken and that a lot more needs to be done before switching is well understood.

\section{REFERENCES}

[1] M. Aguiar, Vector Fields with heteroclinic networks, PhD Thesis, Departamento de Matemática Aplicada, Faculdade de Ciências da Universidade do Porto, 2003

[2] M. Aguiar, P. Ashwin, A. Dias and M. Field, Robust heteroclinic cycles in coupled cell systems: Identical cells with asymmetric inputs, CMUP preprint 2008-06

[3] M. Aguiar, S. B. Castro and I. S. Labouriau, Simple Vector Fields with Complex Behaviour, Int. Jour. of Bifurcation and Chaos, Vol. 16, Nr.2, 2006

[4] M. Aguiar, S. B. Castro and I. S. Labouriau, Dynamics near a heteroclinic network, Nonlinearity 18, 2005

[5] D. Armbruster, E. Stone and V. Kirk, Noisy heteroclinic networks, Chaos, Vol. 13, Nr.1, March 2003

[6] P. Ashwin and M. Field, Heteroclinic Networks in Coupled Cell Systems Arch. Rational Mech. Anal., Vol. 148, 1999, pages 107-143

[7] W. Brannath, Heteroclinic networks on the tetrahedron, Nonlinearity, Vol. 7, 1994, pages $1367-1384$

[8] M. Field, Lectures on bifurcations, dynamics and symmetry, Pitman Research Notes in Mathematics Series, vol. 356, Longman, 1996 
[9] M. I. Golubitsky, I. Stewart, and D. G. Schaeffer, Singularities and Groups in Bifurcation Theory, Vol. II, Springer, 2000

[10] J. Guckenheimer and P. Worfolk, Instant Chaos, Nonlinearity, Vol. 5, 1992, pages $1211-1222$

[11] J. Hofbauer, Heteroclinic Cycles in Ecological Differential Equations, Tatra Mountains Math. Publ., Vol. 4, 1994, pages 105-116,

[12] J. Hofbauer, Heteroclinic Cycles on the simplex, Proc. Int. Conf. Nonlinear Oscillations, Janos Bolyai Math. Soc. Budapest, 1987

[13] J. Hofbauer and K. Sigmund, The Theory of Evolution and Dynamical Systems, Cambridge University Press, Cambridge, 1988

[14] V. Kirk and A. M. Rucklidge The effect of symmetry breaking on the dynamics near a structurally stable heteroclinic cycle between equilibria and a periodic orbit, Dynamical Systems: An International Journal, 23, 2008, pages 42-74

[15] V. Kirk and M. Silber, A competition between heteroclinic cycles, Nonlinearity, Vol. 7, 1994, pages 1605-1621

[16] M. Krupa, Robust Heteroclinic Cycles, J. Nonlinear Sci., 7, 1997, pages 129176

[17] M. Krupa, and I. Melbourne, Asymptotic Stability of Heteroclinic Cycles in Systems with Symmetry, Ergodic Theory and Dynam. Sys., Vol. 15, 1995, pages $121-147$

[18] M. Krupa and I. Melbourne, Asymptotic Stability of Heteroclinic Cycles in Systems with Symmetry, II, Proc. Roy. Soc. Edinburgh, 134A, 2004, pages 1177-1197

[19] I. Melbourne, An example of a non-asymptotically stable attractor Nonlinearity, Vol. 4, 1991, pages 835-844

[20] I. Melbourne, M. R. E. Proctor and A. M. Rucklidge, A heteroclinic model of geodynamo reversals and excursions Dynamo and Dynamics, a Mathematical Challenge (eds. P. Chossat, D. Armbruster and I. Oprea, Kluwer: Dordrecht, 2001, pages $363-370$

[21] C. M. Postlethwaite and J. H. P. Dawes, Regular and irregular cycling near a heteroclinic network Nonlinearity, Vol. 18, 2005, pages 1477-1509

[22] V. S. Samovol, Linearization of a system of differential equations in the neighbourhood of a singular point, Sov. Math. Dokl, Vol. 13, 1972, pages 1255-1959

[23] Y. Sato, E. Akiyama and J. P. Crutchfield, Stability and diversity in collective adaptation, Physica D, Vol. 210, 2005, pages 21-57

[24] T. Ura, On the flow outside a closed invariant set: stability, relative stability and saddle sets, Contributions to Differential Equations, vol. III, No. 3, 1964, pages 249-94

(M.A.D.Aguiar) Centro de Matemática da Universidade do Porto, and Faculdade de Economia da Universidade do Porto, Rua Dr. Roberto Frias, 4200-464 Porto, Portugal

E-mail address: maguiar@fep.up.pt

(I.S. Labouriau and A.A.P. Rodrigues) Centro de Matemática da Universidade do Porto, and Faculdade de Ciências da Universidade do Porto, Rua do Campo Alegre 687, 4169-007 Porto, Portugal

E-mail address, I.S.Labouriau: islabour@fc.up.pt

E-mail address, A.A.P.Rodrigues: alexandre.rodrigues@fc.up.pt 